\title{
Importance of patients' care after COVID-19 positive test
}

\author{
Fanta SA Yadang, Gabriel A Agbor \\ Centre for Research on Medicinal Plants and Traditional Medicine, Institute of Research and Medicinal \\ Plants Studies, PO box 13033, Yaoundé, Cameroon \\ Correspondence: Gabriel A Agbor, agogae@yahoo.fr
}

Received: 07/07/2021
Accepted: 23/08/2021
DOI: https://dx.doi.org/10.4314/jcas.v17i1.5
C The Authors. This work is published under the Creative Commons Attribution 4.0 International Licence.

\section{Introduction}

The emergence of the novel Coronavirus disease (COVID-19) in December 2019 in Wuhan, China, has changed the live style of many people and healthcare systems around the world. Since 11th March 2020, when WHO declared COVID-19 as a global pandemic (WHO 2020a), millions of cases have been detected and millions of deaths registered touching all the continents. After a COVID-19 positive test, the attention of the health care provider and patient's family is mostly directed on the COVID-19 disease while they ignore other health complications which may sometimes result to death of a patient. Most people with COVID-19 develop mild or uncomplicated illness that can be managed at the primary health care level but these are often ignored when a patient test positive for COVID19 which may be fatal to the patient. WHO has established that, primary health care is an essential foundation for the global response to coronavirus disease 2019 (COVID-19) (WHO 2020b). This is because Primary care plays a significant role in gatekeeping and clinical responses by differentiating patients with respiratory symptoms from those with COVID-19. Hence making an early diagnosis and aid vulnerable people cope with their anxiety about the virus (WHO 2020b). Hence, primary care can play a significant role in the COVID-19 response. However, all these may not be effective, as fear, stigmatisation and other health condition pose a serious problem in care of patients with positive test of COVID-19.

\section{Fear and Stigmatisation as a barrier of COVID-19 patients care}

During the early months of the pandemic, fear about the novel disease and death, misinformation, lack of knowledge about COVID-19, identification of infectious clusters, spreaders, or community outbreaks caused widespread fear, panic and anxiety across the globe (Sanjeet, 2020; Mahmud and Islam, 2020). Stigmatisation is a mark of disgrace that sets a person or a group of persons apart from others (Neuberg et al., 2000; Pescosolido, 2003; Ramaci et al., 2020). Social stigma has a variety of negative consequences that lead to reduction of patients seeking care or recovery, reduction in people adhering to interventions, including selfisolation (Giorgi et al., 2019). With the outbreak of the COVID-19 pandemic, people who are infected and/or suspected with symptoms are labelled, stereotyped, and discriminated against because of a perceived link (Ramaci et al., 2020). Therefore, COVID-19 has been associated with many elements of social stigma and fear. Once a 
people avoid contact with such. Caring for the infected person become difficult, as people are afraid of approaching them because of the risk of contracting the deadly disease. At such, the hospital ward is deserted, if not the hospital just because someone tested positive there. Those who are supposed to take care of the patient avoid the patient and such patients become at risk of death. A study from Vietnam found that $18 \%$ of healthcare workers felt unsafe to work in healthcare facilities after they undertook quarantine due to a contact with a COIVID-19 patient (Do Duy et al., 2020). A meta-analysis study on COVID-19 comorbidities has shown that the most common comorbidities identified in a total of 1786 patients from January 24 through march 16, 2020 were hypertension $(15.8 \%)$, cardiovascular and cerebrovascular conditions (11.7\%), and diabetes (9.4\%) (Paudel et al., 2020, Sanyaolu et al., 2020). The review articles of Stefan (Stefan et al., 2021) and Flaherty (Flaherty et al., 2020) reported that patients with pre-existing comorbidities are at greater risk of developing severe and fatal COVID-19 disease. Such lack of patient care, discrimination and stigmatization induce fear and push patients to hide their illness leading to severe complications for those with underlying health conditions or comorbidities and to the spreading of the virus (Sanyaolu et al. 2020).

WHO, reported that fear can also hold people back from seeking immediate health care aggravating their health condition leading sometimes to death. Sotgiu, and Dobler (2020) reported that, anxiety and fear of COVID-19 patients to being discriminated by the community can lead to two clinical and public health consequences: delayed presentation of symptomatic patients to healthcare services and under detection of infectious individuals (increased viral transmission to susceptible contacts). A delayed diagnosis has been associated with more severe disease, mainly in the elderly and in vulnerable groups, while a delayed notification of an infectious patient can facilitate the rapid spread of SARS-CoV-2 in the community (Rong et al., 2020). Also, a late health care of COVID-19 patients leads to severe complication of the disease increasing the death rate of this pandemic. Fear to be stigmatized may keep patient at home from seeking primary care and disease management they need.

\section{Comorbidities}

Comorbidity is defined as the presence of one or more additional medical conditions often occurring or co-existing with the primary condition (Lanmed, 2020). In the context of the COVID19 pandemic, it refers to existing chronic diseases leading people at the higher risk of developing complication if they are infected by COVID-19 (Lanmed, 2020). For an effective health care of COVID-19 patients, a full check-up of the medical conditions of patients should be done before the prescription of any medication to avoid other complications related to comorbidities that the patients could have. Chronic medical conditions affecting the clinical course of COVID19. An overall observation showed that the leading comorbid conditions include hypertension, diabetes, cardiovascular and cerebrovascular diseases, and diabetes. People with underlying uncontrolled medical conditions are at increased risk of COVID-19 infection (CDC, 2020). In the majority of the cases, the management of the first symptoms of COVID-19 is vital for the life of the patients. But a special attention should be given to patients with comorbidities. As it has been reported in literature, patients with preexisting cardiovascular diseases appear to have heightened vulnerability to develop COVID-19 complications and tend to have more severe disease with worse clinical outcomes (Bansal, 2020). The incubating period of SARS-COV-2 is 2 to 14 days (CDC, 2020). During this period, 
focusing only on the COVID-19 symptoms can be detrimental for the patient with comorbidities. There are already some pieces of evidence that COVID-19 can cause damage to tissues and organs other than the lungs and enhance the inflammatory cytokine release which is a vital factor that aggravates disease progression (Wan et al., 2020). A higher level of IL-6 and IL-10, and lower levels of $\mathrm{CD} 4+\mathrm{T}$ and $\mathrm{CD} 8+\mathrm{T}$ are observed in COVID-19 patients parallel with the severity of the disease (Wan et al., 2020). After having recovered from COVID-19, it's important to follow a healthy lifestyle and also take note of alarming signals. It is important to manage well the comorbidities of COVID-19 patients to reduce the severity of the disease.

\section{Conclusion}

For the well-being of COVID-19 patients, it's important to reduce the impact of social stigma, fear and anxiety related to this pandemic by giving the right information concerning how COVID19 spreads and rebuilding patient confidence and hope for life.

\section{References}

Bansal Manish, Cardiovascular disease and COVID-19. Diabetes Metab Syndr., 2020, 14(3): 247-250.

CDC. Coronavirus (COVID-19): symptoms of coronavirus. Centers for Disease Control and Prevention. 2020. [Accessed April 18, 2020, https://www.cdc.gov/coronavirus/2019-ncov/ symptoms-testing/symptoms.html].

Do Duy C, Nong VM, Van AN, Thu TD, Do Thu N, Quang TN. COVID-19 related stigma and its association with mental health of health-care workers after quarantined in Vietnam. Psychiatry Clin Neurosci. 2020;74(10):566-8. https:// doi.org/10.1111/pcn.13120.
Flaherty, G.T., Hession, P., Liew, C.H. et al. COVID-19 in adult patients with pre-existing chronic cardiac, respiratory and metabolic disease: a critical literature review with clinical recommendations. Trop Dis Travel Med Vaccines 6, 16 (2020). https://doi.org/10.1186/s40794020-00118-y

Giorgi, G.; Arcangeli, G.; Ariza-Montes, A.; Rapisarda, V.; Mucci, N. Work-related stress in the Italian banking population and its association with recovery experience. IJOMEH 2019, 32, 255-265.

Lenmed. Everything You Want to Know About Comorbidities and COVID-19. https:// www.lenmed.co.za/ everything-you-want-toknow-about-comorbidities-and-COVID-19/

Mahmud Ashek, Islam M. Rezaul, 2020. Social Stigma as a Barrier to COVID-19 Responses to Community Well-Being in Bangladesh. International Journal of Community Well-Being. https://doi.org/10.1007/s42413-020-00071-w

Neuberg, S. L., Smith, D. M., \& Asher, T., 2000. Why people stigmatize: Toward a biocultural framework. In T. F. Heatherton, R. E. Kleck, M. R. Hebl, \& J. G. Hull (Eds.), The social psychology of stigma (pp. 31-61). New York: Guilford Press.

Paudel S.S. A meta-analysis of 2019 novel coronavirus patient clinical characteristics and comorbidities. Research Square. 2020. 10.21203/rs.3.rs-21831/v1 [Accessed April 18, 2020, https://www.researchsquare.com/article/ rs-21831/v1].

Pescosolido, B.A. The public stigma of mental illness: What do we think; what do we know; what can we prove? J. Health Soc. Behav 2013, $54,1-21$. 
Ramaci, T., Barattucci, M., Ledda, C., \& Rapisarda, V. (2020). Social Stigma during COVID-19 and its impact on HCWs outcomes. Sustainability, 12(9), 3834.

Rong XM, Yang L, Chu HD, Fan M. Effect of delay in diagnosis on transmission of COVID19. Math Biosci Eng. 2020;17(3):2725-2740.

Sanjeet Bagcchi, Stigma during the COVID-19 pandemic. Healthcare workers and patients who have survived COVID-19 are facing stigma and discrimination all over the world. The Lancet, Vol 20 July 2020.

Sotgiu G, Dobler CC. Social stigma in the time of Coronavirus. Eur Respir J, 2020; in press (https://doi.org/10.1183/13993003.024612020)

Stefan, N., Birkenfeld, A.L. \& Schulze, M.B. Global pandemics interconnected — obesity, impaired metabolic health and COVID-19. Nat Rev Endocrinol 17, 135-149 (2021). https:// doi.org/10.1038/s41574-020-00462-1
Wan S, Yi Q, Fan S, Lv J, Zhang X, Guo L, et al. Characteristics of lymphocyte subsets and cytokines in peripheral blood of 123 hospitalized patients with 2019 novel coronavirus pneumonia (NCP). medRxiv. 2020:2020.02.10.20021832.

WHO. WHO Director-General's Opening Remarks at the Media Briefing on COVID-19. (2020a). Available online at: https:// www.who.int/dg/speeches/detail/who-directorgeneral-s-opening-remarks-at-the-media-briefingonCOVID-19-11-march-2020 (accessed June 28, 2020).

WHO, Regional Office for the Western Pacific (2020b). Role of primary care in the COVID-1 9 response. Manila: WHO Regional Office for the Western Pacific 